\title{
Original
}

\section{Effects of xylitol-containing chewing gum on the oral microbiota}

\author{
Kenji Takeuchi 1,2), Mikari Asakawa1), Takafumi Hashiba1,3), Toru Takeshita1,2), \\ Youji Saeki'), and Yoshihisa Yamashita1)
}

\author{
1)Section of Preventive and Public Health Dentistry, Division of Oral Health, Growth and Development, \\ Faculty of Dental Science, Kyushu University, Fukuoka, Japan \\ 2)OBT Research Center, Faculty of Dental Science, Kyushu University, Fukuoka, Japan \\ 3)Department of Dentistry, SDF Central Hospital, Tokyo, Japan \\ 4)Oral Science Section, Central Laboratory, Lotte Co., Ltd., Saitama, Japan
}

(Received November 22, 2017; Accepted December 28, 2017)

\begin{abstract}
In this interventional study, a randomized controlled trial was used to evaluate the short-term effects of xylitol-containing chewing gum on the salivary microbiota. In total, 70 healthy adult men recruited from the Japan Ground Self Defense Force participated in the study during a 2-day training at Yamaguchi camp, Yamaguchi Prefecture, Japan. The men were randomly divided into two groups: one group chewed two pieces of xylitol-containing chewing gum 7 times/day for 2 days $(n=34)$ and the other did not $(n=36)$. Baseline and follow-up stimulated saliva samples were collected and the salivary microbial composition was assessed using the $16 \mathrm{~S}$ rRNA gene next-generation sequencing analysis. The total salivary bacterial count was quantified using a quantitative real-time PCR system. No statistically significant difference was found between the two groups regarding any parameter analyzed in the baseline samples; however, the follow-up samples of the test group showed significantly lower total salivary bacterial count than those of the control group. Conversely, no significant difference was observed
\end{abstract}

Correspondence to Dr. Yoshihisa Yamashita, Section of Preventive and Public Health Dentistry, Division of Oral Health, Growth and Development, Faculty of Dental Science, Kyushu University, 3-1-1, Maidashi, Higashi-ku, Fukuoka 812-8582, Japan Fax: +81-92-642-6354 E-mail: yoshi@dent.kyushu-u.ac.jp

J-STAGE Advance Publication: November 15, 2018

doi.org/10.2334/josnusd.17-0446

DN/JST.JSTAGE/josnusd/17-0446 in the overall composition of the salivary microbiota between the baseline and follow-up samples of the two groups. These results indicate that xylitol-containing chewing gum inhibits the increase in total salivary bacteria over a short time during which the salivary microbial composition is not affected.

Keywords: epidemiology; interventional study; oral microbiome; saliva; xylitol.

\section{Introduction}

Xylitol, a naturally occurring five-carbon polyol (sugar alcohol), is used in several products, including chewing gum, as a sugar substitute; it has been shown to have beneficial effects in the maintenance of oral health. Furthermore, xylitol-containing chewing gum decreases the amount of bacteria in saliva (1-4). In a previous randomized, controlled interventional trial study, xylitolcontaining chewing gum reduced the increase in total salivary bacteria during periods of interrupted regular oral hygiene (5). The mechanism of action linking the chewing of xylitol-containing gum and quantity of oral bacteria is not fully understood; however, chewing gum can trap and remove bacteria from the oral cavity (6). Furthermore, xylitol inhibits growth, metabolism, and polysaccharide production in Streptococcus mutans (7-9), a main causative agent of dental caries.

There is growing evidence suggesting that the use of xylitol-containing chewing gum is effective for preventing caries (10-12); however, not much information is avail- 


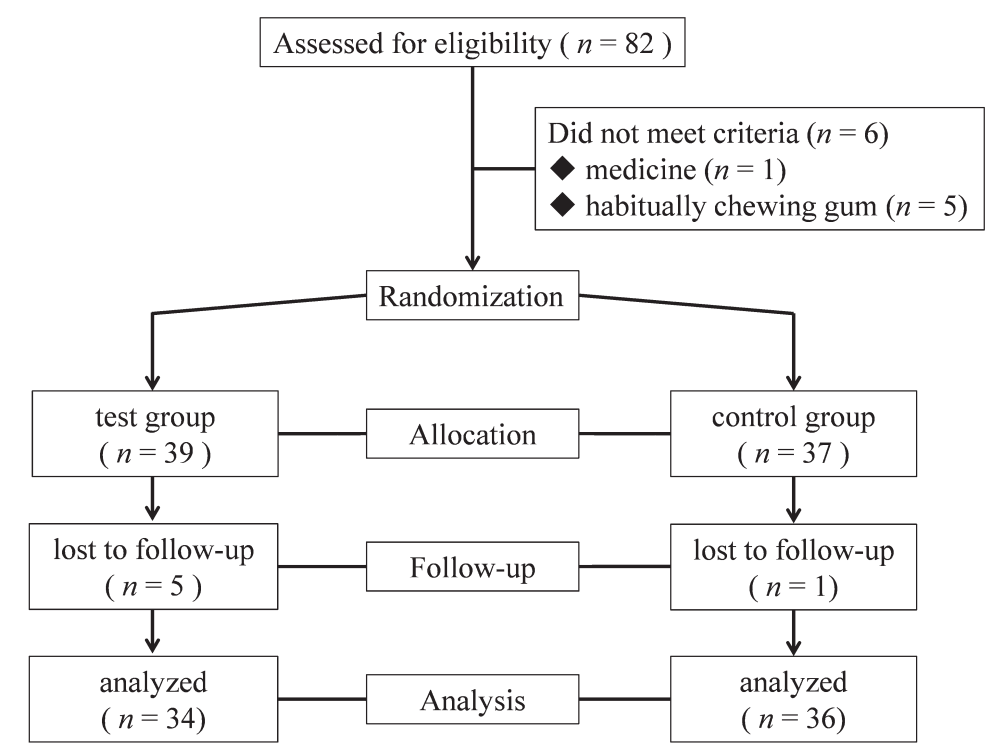

Fig. 1 Study flow diagram.

able about the effects of xylitol-containing chewing gum on the oral microbiota. During the 1990s, the "ecological plaque hypothesis" was widely adopted, which states that microbiota shift from healthy to pathogenic states via bacterial activity and environmental interactions (13). More recently, it has been suggested that microbial consortia, which are aggregations of several different bacteria, can collectively act to initiate and expand caries (14). Soderling et al. (15) demonstrated in a clinical study that the short-term use of xylitol-containing chewing gum does not affect salivary microbial composition in children, but does decrease the salivary $S$. mutans count. However, there have not been any studies examining the effects of xylitol-containing chewing gum on salivary microbial composition in adults. Here, a randomized controlled trial was performed to test the hypothesis that the short-term use of xylitol-containing chewing gum reduces total salivary bacteria count but has no effect on salivary microbial composition in adults.

\section{Materials and Methods}

\section{Study participants}

In May 2015,82 healthy men aged $\geq 20$ years were recruited from the Japan Ground Self Defense Force. The men were scheduled to undergo a 2-day simulated disaster training at Yamaguchi camp, in Yamaguchi Prefecture, Japan. After excluding one individual who was taking antibiotics and five individuals who habitually chewed gum, 76 men were included in the study. All participants provided informed written consent. The study was approved by the Ethics Committee of the faculty of Kyushu University (number 23036) and registered with the UMIN Clinical Trials Registry (UMIN
Clinical Trial Registration No. 000029909). Data were collected, analyzed, and reported in accordance with the Consolidated Standards of Reporting Trials (16).

\section{Sample size calculation}

The sample size was calculated with statistical software (nQuery Advisor, Statistical Solutions, Saugus, MA, USA) using the two-tailed hypothesis test, with a significance level of $5 \%$, power of $80 \%$, and an allocation ratio of $1: 1$. The sample size calculation indicated that if the sample size is $\geq 20$ per group, a significant difference in the total salivary bacteria count, a primary outcome, may be detected.

\section{Study design and intervention}

Figure 1 shows a flow diagram of the study design. The trial protocol was explained to all enrolled participants $(n=76)$ before randomization. After obtaining written informed consent, the commanding officer randomly assigned the participants to either the test group $(n=39)$ or control group $(n=37)$ using a sealed opaque envelope to conceal the random allocations. The examiner and statistician were blinded to the sampling and assignment of groups throughout data collection and analysis. Participants in the test group were instructed to chew commercially available lime-mint flavored xylitol gum (Lotte Co., Ltd., Tokyo, Japan) at a xylitol dose of $7 \mathrm{~g}$ /day for 2 consecutive days during field training, according to the manufacturer's instructions: "Chew two pieces of gum for $5 \min 7$ times a day to promote tooth recalcification." Each piece of gum weighed approximately $1.5 \mathrm{~g}$ and contained $1.16 \mathrm{~g}$ carbohydrates, $0.5 \mathrm{~g}$ xylitol, $0.43 \mathrm{~g}$ maltitol, $0.3 \mathrm{mg}$ phosphate calcium hydrogen, and $1.5 \mathrm{mg}$ funoran 
extract. The xylitol dosage was chosen according to a previous review article showing that 5-10 g/day of xylitol chewed at least thrice a day is effective in reducing the number of oral bacteria (17). Participants in the control group did not chew gum. Stimulated saliva samples were collected the day before the 2-day training (baseline survey) and on the final day of the training (follow-up survey). Due to the nature of simulated disaster training, participants in both groups could not brush their teeth or maintain their oral hygiene during the 2-day training.

\section{Oral examinations and questionnaires}

One month before training, information regarding the oral conditions, hygiene habits, and demographics of the participants was obtained through an oral examination by a dentist and a self-administered questionnaire. All oral examinations were performed by the same dentist. Oral examination data included the number of teeth; an index of decayed, missing, and filled teeth (DMFT); the number of teeth with a pocket depth (PD) of $\geq 4 \mathrm{~mm}$; bleeding upon probing (BOP) as a measure of gingival inflammation (18); and a dental plaque score determined using the Silness and Loe plaque index (19). PD was measured for each tooth using a six-point probing method, excluding the wisdom teeth; the deepest PD was recorded as a periodontal parameter. Examiner reliability was verified by an intraexaminer calibration with volunteers who had similar characteristics to the participants in the study. The resulting Cohen's $\kappa$ value of $>0.8$ indicated a high intraexaminer reliability. Additional information, including smoking history, alcohol consumption, and tooth brushing frequency was obtained from a self-administered questionnaire. Smoking history was classified as current, ever, or non-smoker. Alcohol consumption was classified as every day, sometimes, or almost never. Tooth brushing frequency was classified as once, twice, or thrice a day.

\section{Saliva collection and DNA extraction}

Stimulated saliva sample collection for the baseline and follow-up samples was performed at 11:00 and 12:00 am, respectively. Participants were instructed to refrain from eating, drinking, or tooth brushing for at least $1 \mathrm{~h}$ before sampling. During sampling, participants were instructed to sit in a chair and chew gum free of sweeteners, food additives, and flavoring agents for $3 \mathrm{~min}$. Saliva was then collected in a sterile tube and weighed. The weights were subsequently converted to volumes, standardized to $1 \mathrm{~mL} / \mathrm{g}$ specific gravity. The saliva samples were transported on ice for $2 \mathrm{~h}$ and stored at $-80^{\circ} \mathrm{C}$ until analysis. DNA was extracted from $500 \mu \mathrm{L}$ saliva using a bead- beating method, as previously described (20).

\section{Bacterial quantification via Real-Time PCR}

The total bacterial count per $1 \mathrm{~mL}$ saliva was quantified with a QuantiFast SYBR Green PCR kit (Qiagen, Hilden, Germany) and a StepOne Real-Time PCR System (Applied Biosystems) with the universal bacterial primers 806F (5'-TTA GAT ACC CYG GTA GTC C-3') and 926R (5'-CCG TCA ATT YCT TTG AGT TT-3'), as previously described (21). The $P$. pasteri $16 \mathrm{~S}$ rRNA gene, which was inserted into the pBluescript SK II (+) vector plasmid (Stratagene, La Jolla, CA USA), was used as the real-time control.

\section{Ion Torrent 16S rRNA gene amplicon sequencing}

16S rRNA gene amplicon sequencing analyses were performed as previously described (22). In brief, the $\mathrm{V} 1-\mathrm{V} 2$ regions of the $16 \mathrm{~S}$ rRNA gene in each sample were amplified using the following primers: 8F (5'-AGA GTT TGA TYM TGG CTC AG-3') with the Ion Torrent adaptor $\mathrm{A}$ and a sample-specific 8-base tag sequence, and 338R (5'-TGC TGC CTC CCG TAG GAG T-3') with the Ion Torrent adaptor trP1. Following PCR amplification, purification, and quantification, equal amounts of purified PCR amplicon products were pooled and gel-purified. Emulsion PCR and template-positive particle enrichment were performed using an Ion PGM HiQ OT2 Kit (Life Technologies, Carlsbad, CA, USA) and sequenced using an Ion PGM (Life Technologies) with an Ion PGM HiQ Sequencing Kit (Life Technologies).

Low-quality reads were excluded using a script written in $\mathrm{R}$, according to the following criteria: read length $\leq 200$ bases (not including the tag sequence), average quality score $\leq 25$, correct forward primer sequence not included, correct reverse primer sequence (one mismatch allowed) not included, or the presence of a homopolymer run $>7$ nt. Quality-checked reads were subsequently assigned to their corresponding sample using the tag sequence. Similar sequences were assigned to operational taxonomic units (OTUs) using UPARSE (23), with a minimum pairwise identity of $97 \%$. The taxonomy of the representative sequences was determined using BLAST against 831 oral bacterial $16 \mathrm{~S}$ rRNA gene sequences (HOMD 16S rRNA RefSeq version 13.2) from the Human Oral Microbiome Database (24). Nearestneighbor species with $\geq 98 \%$ identity were selected as candidates for each representative OTU. The taxonomy of the sequences without a hit was determined using RDP classifier, with a minimum support threshold of $80 \%$. The number of OTUs was calculated following rarefaction to 4,000 reads per sample using $R$. 
Table 1 Baseline characteristics of the participants

\begin{tabular}{|c|c|c|c|}
\hline & Control $(n=36)$ & Test $(n=34)$ & $P$-value \\
\hline Age & & & $0.588^{\mathrm{a}}$ \\
\hline median & 27.5 & 27.0 & \\
\hline range & $19.0-52.0$ & $19.0-52.0$ & \\
\hline Number of teeth present & & & $0.881^{\mathrm{a}}$ \\
\hline median & 29.0 & 29.0 & \\
\hline range & $18.0-32.0$ & $23.0-32.0$ & \\
\hline DMFT & & & $0.524^{\mathrm{a}}$ \\
\hline median & 6.0 & 5.5 & \\
\hline range & $0-18.0$ & $0-24.0$ & \\
\hline Number of teeth with $\geq 4 \mathrm{~mm} \mathrm{PD}, \mathrm{N}(\%)$ & & & $0.999^{\mathrm{b}}$ \\
\hline 0 & $28(77.8)$ & $26(76.5)$ & \\
\hline$\geqq 1$ & $8(22.2)$ & $8(23.5)$ & \\
\hline $\mathrm{BOP}(\%)$ & & & $0.934^{\mathrm{a}}$ \\
\hline median & 7.1 & 5.4 & \\
\hline range & $0-87.5$ & $0-71.4$ & \\
\hline Dental plaque score & & & $0.733^{\mathrm{a}}$ \\
\hline median & 0.3 & 0.4 & \\
\hline range & $0-3.0$ & $0-2.3$ & \\
\hline Smoking history, N (\%) & & & $0.136^{\mathrm{b}}$ \\
\hline current smoker & $19(52.8)$ & $14(41.2)$ & \\
\hline ever smoker & $8(22.2)$ & $4(11.8)$ & \\
\hline non-smoker & $9(25.0)$ & $16(47.1)$ & \\
\hline Alcohol drinking frequency, $\mathrm{N}(\%)$ & & & $0.697^{\mathrm{b}}$ \\
\hline every day & $9(25.0)$ & $8(23.5)$ & \\
\hline sometimes & $25(69.4)$ & $22(64.7)$ & \\
\hline almost never & $2(5.6)$ & $4(11.8)$ & \\
\hline Tooth brushing frequency, $\mathrm{N}(\%)$ & & & $0.894^{\mathrm{b}}$ \\
\hline thrice per day & $5(13.9)$ & $4(11.8)$ & \\
\hline twice per day & $26(72.2)$ & $24(70.6)$ & \\
\hline once per day & $5(13.9)$ & $6(17.6)$ & \\
\hline
\end{tabular}

\section{Statistical analysis}

Demographic differences between control and test groups were determined using Pearson's Chi-square test for categorical variables and Mann-Whitney $U$ test for continuous variables. The total bacterial count or number of OTUs in the saliva samples between the control and test groups was compared using the Mann-Whitney $U$ test. The within-group comparison of the relative abundance of genera in the salivary microbiota between the baseline and follow-up samples was performed using the Wilcoxon signed-rank test. All statistical analyses were performed using R (version 3.1.1), with a significance level of $5 \%$.

\section{Results}

Six study participants failed to take the follow-up survey and were thus excluded from the study. The remaining 70 participants (test group: $n=34$; control group: $n=$ 36) were included in the analysis. The percentage of dropouts was $12.8 \%$ (5/39) for the test group and 2.7\% $(1 / 37)$ for the control group. The reported reason for the dropout of six subjects was that they went separate ways from the group in the middle of the field training. During field training, none of participants brushed their teeth. The mean dose of xylitol was $6.78 \mathrm{~g} /$ day throughout the intervention period.

Table 1 shows the baseline characteristics of the participants. Age, number of teeth present, DMFT, number of teeth with $\geq 4 \mathrm{~mm}$ PD, BOP, dental plaque score, smoking history, alcohol consumption, and tooth brushing frequency did not show a statistically significant difference among the baseline samples obtained from the two groups.

Table 2 shows the total bacterial count or number of OTUs in baseline and follow-up saliva samples for each group. No differences in the total bacterial count or number of OTUs between the two groups were found in the baseline samples. However, the test group follow-up samples showed significantly lower total salivary bacteria counts than the control group follow-up samples $(P=$ 0.004). No difference in the number of OTUs in the saliva samples was found between the groups. A principal coor- 
Table 2 Comparison of total bacterial count or number of OTUs in the saliva samples between control and test groups

\begin{tabular}{|c|c|c|c|c|c|c|}
\hline & \multicolumn{3}{|c|}{ Baseline } & \multicolumn{3}{|c|}{ Follow-up } \\
\hline & median & range & $P$-value & median & range & $P$-value \\
\hline \multicolumn{7}{|c|}{ Total bacterial count $\left(\log _{10}\right.$ copies $\left./ \mathrm{mL}\right)$} \\
\hline control group & 8.4 & 7.5-8.9 & 0.681 & 8.7 & $8.1-9.7$ & 0.004 \\
\hline test group & 8.4 & $8.0-8.9$ & & 8.5 & $7.5-9.1$ & \\
\hline \multicolumn{7}{|l|}{ Number of OTUs } \\
\hline control group & 170 & $125-214$ & 0.698 & 166 & $119-204$ & 0.109 \\
\hline test group & 172 & $132-214$ & & 173 & $114-208$ & \\
\hline
\end{tabular}

OTUs, operational taxonomic units.
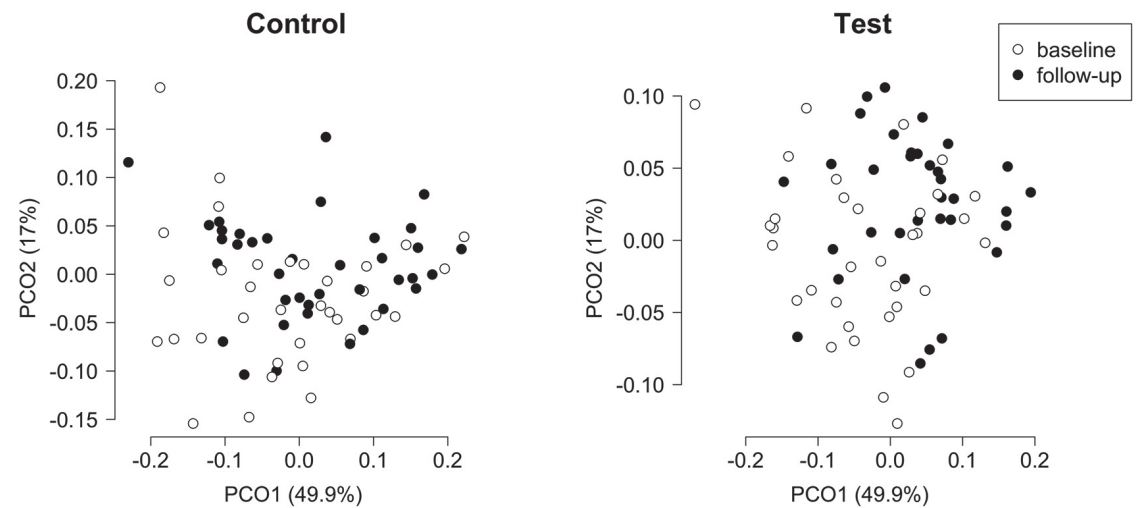

Fig. 2 Principal coordinate analysis plots showing similarities in the salivary microbiota between the baseline and follow-up samples [left, control group $(n=36)$; right, test group $(n=34)$ ]. Plots were generated using a weighted UniFrac distance metric. The two components explain $69.3 \%$ of the variance. PCO, principal coordinate.
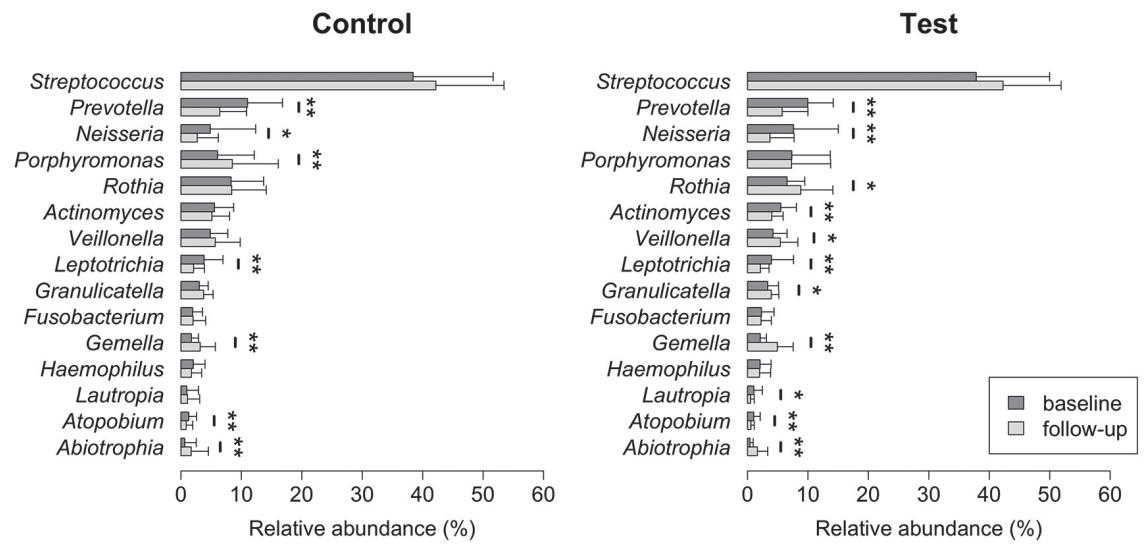

Fig. 3 Mean relative abundances of dominant bacterial genera in the salivary samples. Only genera with a mean relative abundance of at least $1 \%$ in each group are shown. ${ }^{* *} P$ $<0.01 ; * P<0.05$.

dinate analysis plot, based on weighted UniFrac, found no difference in the overall composition of the salivary microbiota between the baseline and follow-up samples in both control and test groups (Fig. 2). Furthermore, the salivary microbial composition in the baseline samples did not differ between the two groups.

Figure 3 shows the mean relative abundances of the dominant bacterial genera in each of the two groups. In the baseline samples of both the groups, Streptococcus was the most predominant genus. The genera Prevotella,
Neisseria, Porphyromonas, and Rothia were also present in high proportions. Significant differences were found between the baseline and follow-up samples in mean relative abundance of some genera, including Prevotella and Neisseria. However, an abundance shift specific to the test group was not observed.

\section{Discussion}

This interventional study demonstrated that short-term use of xylitol-containing chewing gum inhibits the 
increase in total salivary bacteria quantity over a short time of interrupted oral hygiene without affecting salivary microbial composition. Here, we used a randomized controlled trial wherein daily tooth brushing was not performed. To our knowledge, this is the first study to examine the impact of xylitol-containing chewing gum on the bacterial composition of saliva in adults.

The present findings agree with those of Soderling et al. (15) despite differences in methods of detecting bacterial composition and the age group of study participants. Soderling et al. (15) assessed salivary microbial composition in children using a human oral microbe identification microarray and found that apart from a decrease in $S$. mutans count, xylitol-containing chewing gum consumption has no impact on salivary microbial composition. We assessed salivary microbial composition in adults using a barcoded, 16S rRNA pyrosequencing approach, which provides a comprehensive survey of bacterial community composition, including untargeted species. Our results indicate that xylitol gum consumption inhibits the increase in the quantity of total salivary bacteria in the absence of regular oral hygiene, while leaving salivary microbial composition unchanged. The 14 dominant bacterial genera found, including Streptococcus, did not exhibit changes specific to the test group. Furthermore, our results showed that xylitol gum consumption had no significant effect on the number of OTUs in the saliva using a 16S rRNA deep sequencing approach.

Regarding the relative abundance of the dominant bacterial genera in saliva, the results of this study show that change in mean relative abundance in the control group is similar to that of the test group; however, there were significant changes in the mean relative abundances of some genera. Therefore, it is reasonable to assume that shifts in the relative abundance of bacteria in saliva following emergency-preparedness training would be similar to those following natural disasters, wherein daily tooth brushing can be interrupted. Furthermore, the results of this study show that the predominant genus was Streptococcus in both control and test groups and baseline and follow-up samples. These results agree with previous studies, which have found that Streptococcus is a major component of the oral flora (25).

The present study shows that xylitol-containing chewing gum suppressed the increase in the number of total salivary bacteria in the study participants compared with those who did not chew gum. These results are supported by previous studies, which reported similar findings $(6,26-29)$. Furthermore, in a previous interventional study, which also used an exploratory randomized, controlled trial design, we found significant suppressive effects of the same xylitol-containing gum as used in this study (5).

Xylitol-containing chewing gum has been shown to reduce the formation of oral biofilms on tooth surfaces $(27,28)$. Furthermore, the number of total salivary bacteria is known to be positively correlated with the amount of tooth surface plaque $(26,29)$. Moreover, a previously described mechanism linking xylitol to the prevention of cavities caused by oral bacteria is supported by the results of this study (i.e., a decrease in biofilm formation results from inhibition of the increase in oral bacteria count). Therefore, our results examining total salivary bacteria quantity partially reflect the oral hygiene status of the participants and suggest that the use of xylitol-containing chewing gum may be a useful oral cleaning tool for preventing tooth decay and maintaining good oral hygiene.

Results from this study also suggest that the use of xylitol gum may be helpful in maintaining oral health during disasters, wherein regular oral hygiene practices may be interrupted. It has been reported that people in areas affected by earthquakes and tsunamis find it difficult to maintain daily oral hygiene due to a lack of water and materials necessary for oral hygiene, including toothbrushes and toothpaste (30). Since xylitol-containing chewing gum inhibits the increase in salivary bacteria without change in oral microbial composition, even in the absence of water and oral hygiene products, its use may help maintain oral health under conditions that interfere with regular oral hygiene care.

There are potential limitations to this study. First is the short 2-day intervention period. Unfortunately, it was unable to extend the intervention period because the trial period was dictated by the field training schedule. Therefore, a trial examining the chewing of gum for prolonged periods is necessary to determine the long-term effects of xylitol-containing chewing gum on the salivary microbiota.

In conclusion, the present study indicates that shortterm consumption of commercial xylitol gum has an inhibitory effect on salivary bacterial growth without affecting salivary microbial composition in adults. These findings suggest that chewing xylitol gum may be helpful for preventing oral diseases, including dental caries, and in maintaining oral health under conditions that make regular daily oral hygiene difficult.

\section{Acknowledgments}

We are grateful to the subjects who participated in this study. This study was funded by Lotte Co., Ltd. 


\section{Conflict of interest}

Y. Saeki contributed to the study while employed by Lotte Co., Ltd. The sponsor had no control over the interpretation of the results or publication of this study.

\section{References}

1. Ly KA, Milgrom P, Roberts MC, Yamaguchi DK, Rothen M, Mueller G (2006) Linear response of mutans streptococci to increasing frequency of xylitol chewing gum use: a randomized controlled trial. BMC Oral Health 6, 6.

2. Nakai Y, Shinga-Ishihara C, Kaji M, Moriya K, MurakamiYamanaka K, Takimura M (2010) Xylitol gum and maternal transmission of mutans streptococci. J Dent Res 89, 56-60.

3. Seki M, Karakama F, Kawato T, Tanaka H, Saeki Y, Yamashita Y (2011) Effect of xylitol gum on the level of oral mutans streptococci of preschoolers: block-randomized trial. Int Dent J 61, 274-280.

4. Shinga-Ishihara C, Nakai Y, Milgrom P, Söderling E, Tolvanen M, Murakami K (2012) Xylitol carryover effects on salivary mutans streptococci after 13 months of chewing xylitol gum. Caries Res 46, 519-522.

5. Hashiba T, Takeuchi K, Shimazaki Y, Takeshita T, Yamashita Y (2015) Chewing xylitol gum improves self-rated and objective indicators of oral health status under conditions interrupting regular oral hygiene. Tohoku J Exp Med 235, 39-46.

6. Wessel SW, van der Mei HC, Morando D, Slomp AM, van de Belt-Gritter B, Maitra A et al. (2015) Quantification and qualification of bacteria trapped in chewed gum. PLoS One 10, e0117191.

7. Söderling E, Alaräisänen L, Scheinin A, Mäkinen KK (1987) Effect of xylitol and sorbitol on polysaccharide production by and adhesive properties of Streptococcus mutans. Caries Res 21, 109-116.

8. Söderling EM, Ekman TC, Taipale TJ (2008) Growth inhibition of Streptococcus mutans with low xylitol concentrations. Curr Microbiol 56, 382-385.

9. Söderling EM (2009) Xylitol, mutans streptococci, and dental plaque. Advances in Adv Dent Res 21, 74-78.

10. Isokangas P, Alanen P, Tiekso J, Makinen KK (1988) Xylitol chewing gum in caries prevention: a field study in children. Am Dent Assoc 117, 315-320.

11. Burt BA (2006) The use of sorbitol- and xylitol-sweetened chewing gum in caries control. J Am Dent Assoc 137, 190-196.

12. Campus G, Cagetti M, Sale S, Petruzzi M, Solinas G, Strohmenger L et al. (2013) Six months of high-dose xylitol in high-risk caries subjects--a 2-year randomized, clinical trial. Clin Oral Investig 17, 785-791.

13. Marsh PD (1994) Microbial ecology of dental plaque and its significance in health and disease. Adv Dent Res Jul 8, 263-271.

14. Simon-Soro A, Mira A (2015) Solving the etiology of dental caries. Trends Microbiol 23, 76-82.
15. Söderling E, ElSalhy M, Honkala E, Fontana M, Flannagan S, Eckert G et al. (2015) Effects of short-term xylitol gum chewing on the oral microbiome. Clin Oral Investig 19, 237-244.

16. Schulz KF, Altman DG, Moher D; CONSORT Group (2010) CONSORT 2010 statement: updated guidelines for reporting parallel group randomized trials. Ann Intern Med 152, 726-732.

17. Ly KA, Milgrom P, Rothen M (2008) The potential of dentalprotective chewing gum in oral health interventions. J Am Dent Assoc 139, 553-563.

18. Lang NP, Joss A, Orsanic T, Gusberti FA, Siegrist BE (1986) Bleeding on probing. A predictor for the progression of periodontal disease? J Clin Periodontol 13, 590-596.

19. Löe H (1967) The gingival index, the plaque index and the retention index systems. J Periodontol 38, 610-616.

20. Yamanaka W, Takeshita T, Shibata Y, Matsuo K, Eshima N, Yokoyama T et al. (2012) Compositional stability of a salivary bacterial population against supragingival microbiota shift following periodontal therapy. PLoS One 7, e42806.

21. Takeshita T, Nakano Y, Kumagai T, Yasui M, Kamio N, Shibata Y et al. (2009) The ecological proportion of indigenous bacterial populations in saliva is correlated with oral health status. ISME J 3, 65-78.

22. Kageyama S, Takeshita T, Asakawa M, Shibata Y, Takeuchi K, Yamanaka W et al. (2017) Relative abundance of total subgingival plaque-specific bacteria in salivary microbiota reflects the overall periodontal condition in patients with periodontitis. PLoS One 12, e0174782.

23. Edgar RC (2013) UPARSE: highly accurate OTU sequences from microbial amplicon reads. Nat Methods 10, 996-998.

24. Chen T, Yu WH, Izard J, Baranova OV, Lakshmanan A, Dewhirst FE (2010) The human oral microbiota database: a web accessible resource for investigating oral microbe taxonomic and genomic information. Database (Oxford) 2010, baq013.

25. Dewhirst FE, Chen T, Izard J, Paster BJ, Tanner AC, Yu WH et al. (2010) The human oral microbiome. J Bacteriol 192, 5002-5017.

26. Schaeken MJ, Creugers TJ, Van der Hoeven JS (1987) Relationship between dental plaque indices and bacteria in dental plaque and those in saliva. J Dent Res 66, 1499-1502.

27. Birkhed D (1994) Cariologic aspects of xylitol and its use in chewing gum: a review. Acta Odontol Scand 52, 116-127.

28. Milgrom P, Ly KA, Roberts MC, Rothen M, Mueller G, Yamaguchi DK (2006) Mutans streptococci dose response to xylitol chewing gum. J Dent Res 85, 177-181.

29. Price RR, Viscount HB, Stanley MC, Leung KP (2007) Targeted profiling of oral bacteria in human saliva and in vitro biofilms with quantitative real-time PCR. Biofouling 23, 203-213.

30. Hosokawa R, Taura K, Ito E, Koseki T (2012) Roles of dentists and dental hygienists in two major earthquakes. Int Dent J 62, 315-319. 